Journal of Engineering and Applied Sciences 14 (Special Issue 2): 5460-5465, 2019

ISSN: 1816-949X

(C) Medwell Journals, 2019

\title{
Some Optical Properties of Synthesized Graphene Oxide Hybrid Cupper Phthalocyanine
}

\author{
Saad Abbas Jasim and Raheem Gaayid Kadhim \\ Department of Physics, College of Science, University of Babylon, Babylon, Iraq \\ Saad.abbas1988@gmail.com
}

\begin{abstract}
Graphene oxide samples were prepared by modified Hummer's method and the samples were investigated structurally and chemically by X-Ray Diffraction (XRD), Atomic Force Microscopy (AFM) and Fourier Transform Infrared spectroscopy (FTIR). The optical properties of polymer composite GO-CuPc (Graphene Oxide-Cupper Phthalocyanines) prepared by spin coating technique were studied. Three ratio of graphene oxide added to the pure cupper phthalocyanines (10,15 and 20\%). The tests of UV-VIS spectrometer of the wavelength range for which absorption spectra have been recorded is $300-900 \mathrm{~nm}$. For indirect allowed and forbidden transition the absorption coefficient, extinction coefficient and energy gap have been determined. Results show an increasing in absorption coefficient and extinction coefficient and decreasing in the energy gap with the ratio of graphene oxide.
\end{abstract}

Key words: FTIR, GO-CuPc, cupper phthalocyanines, forbidden transition, absorption coefficient, extinction coefficient

\section{INTRODUCTION}

Graphene-based materials are widely applied for technological applications in many fields, especially in elastic, flexible electron-ics where individual nanocarbon objects such as single graphene flakes (Ahn and Je, 2012) and/or carbon nanotubes (Hsiou et al., 2004) play the crucial role. The number of methods for a large scale graphene production has been already employed. These methods involve: mechanical cleav-ing, epitaxial growth, chemical vapor deposition, tearing of carbon nanotubes and reduction of produced Graphene Oxide (GO) from graphite and/or exfoliated graphite, direct exfoliation of graphite in a solvent. The most commonly applied the modified Hummer's methods (Hummers and Offeman, 1958). Graphene, a single layer of carbon atoms bonded into a honeycomb two-dimensional lattice has attracted great interests from scientists and engineers because of its extraordinary properties and wide applications (Choi and Lee, 2012; Eigler and Hirsch, 2014) such as functional films, electric devices and energy storage devices (Li-ion batteries, supercapacitors) etc. Different from graphene which is almost not soluble and cannot be dispersed in water or any organic solvent (Eigler and Hirsch, 2014; Spitalsky et al., 2011), Graphene Oxide (GO) contains high-density oxygen functional groups, like hydroxyl and epoxy group on its basal plane and carboxyl at its edge. They afford GO with excellent water solubility, ease of functionalization and convenience in processing etc., making it the most popular precursor of graphene. Undoubtedly, it is of great significance to develop economical, eco-friendly and scalable routines to produce GO (Dimiev and Tour, 2014; Shamaila et al., 2016).

Phthalocyanine derivatives which have a similar structure to porphyrin have been utilized in important functional materials in many fields. Their useful properties are attributed to their efficient electron transfer abilities. The central cavity of phthalocyanines is known to be capable of accomodating 63 different elemental ions including hydrogens (metal-free Phthalocyanine, H2-PC). A phthalocyanine containing one or two metal ions is called a Metal Phthalocyanine (M-PC). In the last decade, as a result of their high electron transfer abilities, M-PCs have been utilized in many fields such as molecular electronics, optelectronics, photonics, etc. (Sakamoto et al., 2004; De La Torre et al., 2004).

In the present research synthesis of graphene oxide by modified Hummer's method have been described, the prepared samples was investigate structurally and chemically by X-ray diffraction, scanning electron microscope, FTIR and atomic force microscopy after that three different ratio of graphene oxide was added to nickle phthalocyanine, then some optical properties have been studied.

Corresponding Author: Saad Abbas Jasim, Department of Physics, College of Science, University of Babylon, Babylon, Iraq, Saad.abbas1988@gmail.com 


\section{MATERIALS AND METHODS}

\section{Experimental part}

Required materials: Graphite flakes (acid treated 99\%), potassium permanganate (99\%), phosphoric acid (99\%), hydrogen peroxide (98\%), sulphuric acid (98\%) hydrochloric acid (35\%), nickle phthaylocinine.

Graphene oxide syntheised by Hummer's method: Graphene oxide prepared by using modified Hummer's method which is in detail, $1 \mathrm{~g}$ of graphite flake added to $6 \mathrm{~g}$ of Potassium permanganate then adding $120 \mathrm{~mL}$ of sulphuric acid and $13.3 \mathrm{~g}$ of phosphoric acid, the mixture was heated nd stirred at 50 celsius for $12-14 \mathrm{~h}$ at the start the colour of the mixture is green and after $12 \mathrm{~h}$ it become brown, for purification the mixture was washed by centrifugation and rinsing with $8 \%$ hydrochloric acid and (DI) water for various time after that the sample was dried by hot air oven, the graphene oxide optained as a powder. After that graphene oxide was desolved in DMF, films was prepared by drop casting method, three different ratio of graphene oxide was added to Nickle phthaylocinine.

X-Ray Diffraction (XRD): All crystal structures of preparation polymeric nanocomposites have been studied by using the XRD (6000) diffraction device supplied by the Japanese company (SHIMADZU).

Atomic Force Microscope (AFM): Atomic Force Microscope (AFM) micrographs with digital Instruments (Inc. AA2000) are taken to observe the surface roughness and topography of deposited thin films. Typical data have been taken from AFM height images include Root Mean Square (RMS) roughness and grain size. It has three main modes of mapping topography: contact, non-contact and intermittent contact or tapping. The most important part of an AFM is the tip with its nanoscale radius of curvature. The tip is attached to a micronscale cantilever which reacts to the Van der Waals interaction and other forces between the tip and sample.

FTIR measurement: The sample GO are tested Fourier Transfer Infrared Spectroscopy (FTIR) by using Vertex 70 from Shimadzu company.

Optical measurements: The absorption spectrum of $\mathrm{GO}$ have been recorded in the wavelength range $(200-800 \mathrm{~nm})$ by using the double beam spectrophotometer (Shimadzu, UV-1800 $\AA$ ). The absorption spectrum have been recorded at room temperature. A computer program was employed to obtain the optical constants, absorption coefficient, extinction coefficient, refractive index and energy gap.

\section{RESULTS AND DISCUSSION}

$\mathrm{XRD}$ analysis was used to determine the average crystalline properties of the GO sheet. Results are shown in Fig. 1. The GO sheet prepared showed a very strong peak at $2 \theta=10.1^{\circ}$ which is in good agreement with the literatures (Du et al., 2010). The results of XRD initially proved the successful synthesis of GO sheet. The FTIR spectrum Fig. 2 of shows a broad peak between $3000-3700 \mathrm{~cm}^{-1}$ in the high frequency area together with a sharp peak at $1635 \mathrm{~cm}^{-1}$ corresponding to the stretching and bending vibration of $\mathrm{OH}$ groups of water molecules adsorbed on graphene oxide. Therefore, it can be concluded that the sample has strong hydrophilicity. The absorption peaks at 2930 and $2850 \mathrm{~cm}^{-1}$ are represent the symmetric and anti-symmetric stretching vibrations of

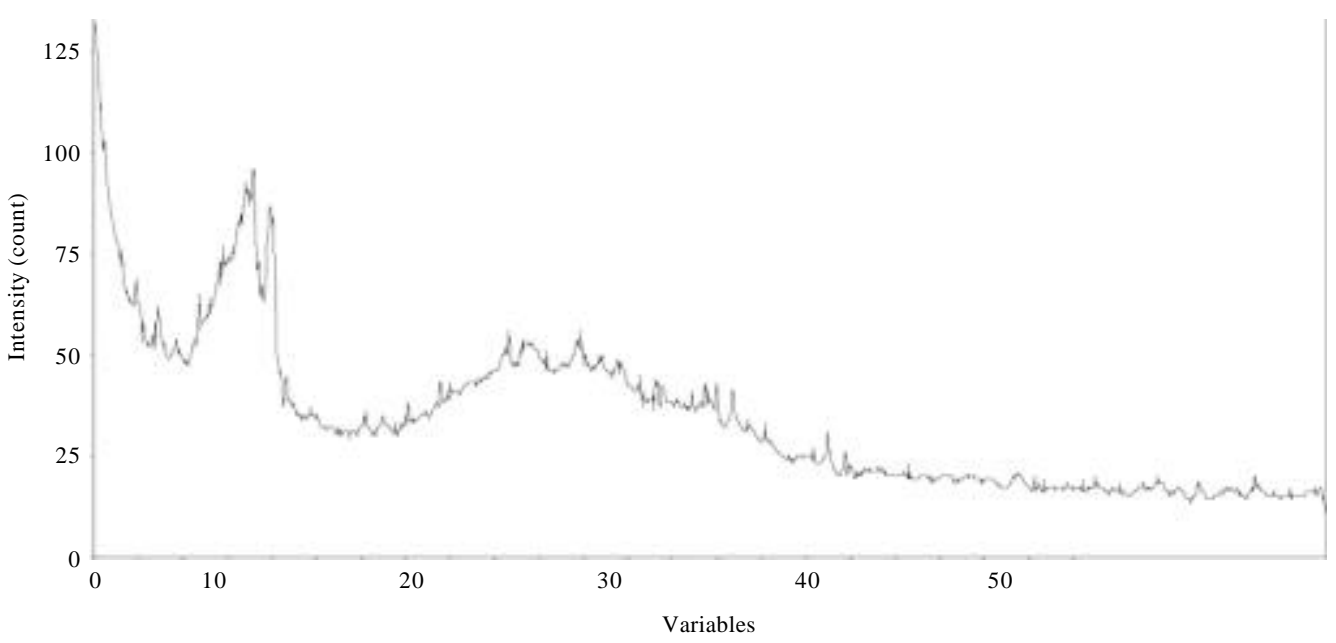

Fig. 1: XRD of graphene oxide 


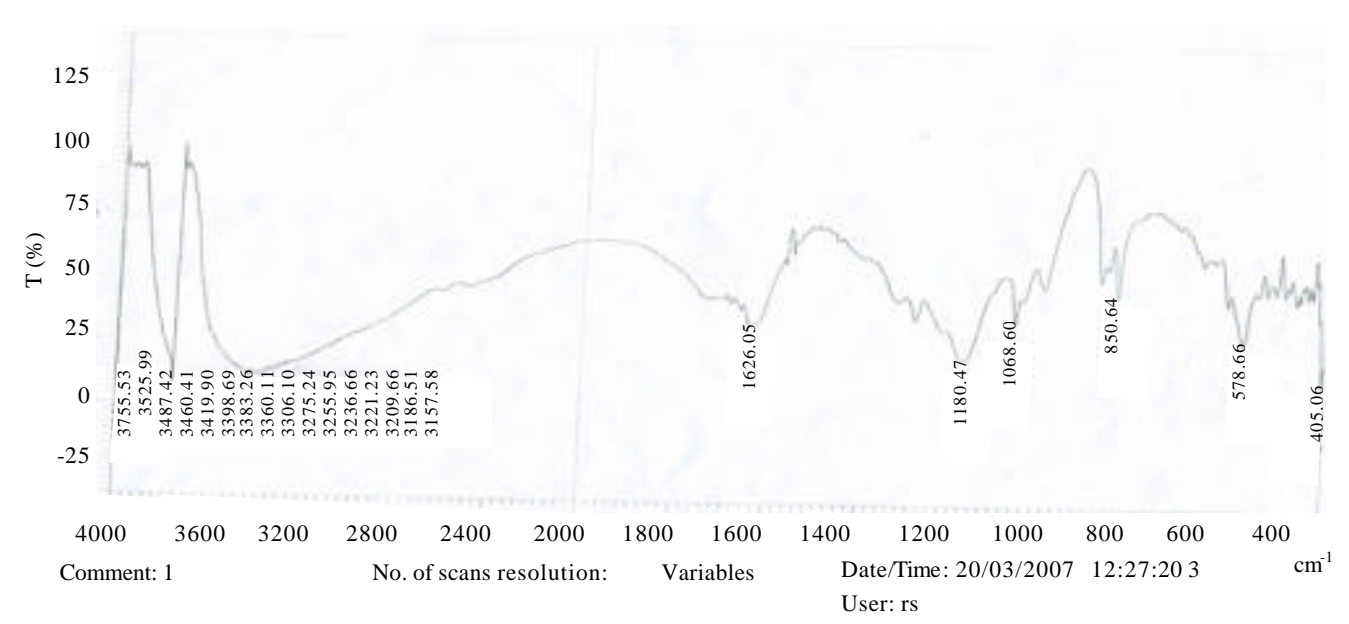

Fig. 2: FT-IR patters of prepared graphene oxide

$\mathrm{CH}_{2}$ while the presence of two absorption peaks observed in the medium frequency area at 1630 and $740 \mathrm{~cm}^{-1}$ can be attributed to the stretching vibration of $\mathrm{C}=\mathrm{C}$ and $\mathrm{C}=\mathrm{O}$ of carboxylic acid and carbonyl groups present at the edges of graphene oxide. Finally, the absorption peaks at 1385 and $1110 \mathrm{~cm}^{-1}$ are correspond to the stretching vibration of $\mathrm{CO}$ of carboxylic acid and $\mathrm{COH}$ of alcohol, respectively. The presence of these oxygen-containing groups reveals that the graphite has been oxidized. The polar groups, especially, the surface hydroxyl groups, result in the formation of hydrogen bonds between graphite and water molecules; this further explains the hydrophilic nature of graphene oxide and this deal with (Shahriary and Athawale, 2014).

To study the surfaces of film materials deposited important to recognize how the distribution and arrangement of atoms on surfaces and get to know the differences or homogeneity properties or attributes relating to each atom separately can through microscopic analysis AFM study the effect of the parameters (thickness, temperature, method of preparation. etc.) on the properties of film material deposited. As well as analysis of the AFM can calculate the thickness of film, roughness and grain size and gives an illustrative picture of the distribution of the particle size of the crystal on the surface rate (Kose et al., 2009).

Figure 3 show three-dimensional, two-dimensional and granularity normal distribution, graphic granular aggregates formed in the film and grain boundaries of GO films. From Fig. 3, it can be observed that GO flakes are typically irregularly shaped. They have a lateral size ranging from few nanometers to some $\mathrm{mm}$, the roughness average $(\mathrm{nm})$ of prepared graphene oxide was $6.02 \mathrm{~nm}$, the root mean square of prepared graphene oxide was $7.5 \mathrm{~nm}$ and the average diameter $(\mathrm{nm})$ of prepared graphene oxide was $48.72 \mathrm{~nm}$, a this deal with (Su et al., 2009).

Figure 4 shows the absorbance of as a function of the wavelength of the incident light for different ratio of graphene oxide added to cupper phtaylocinine. It can be noticed from Fig. 4-8 that the absorbance for all films have decreases with increasing of wavelength. The absorbance of pure $(\mathrm{CuPc})$ and it's additives are increasing with the increasing of the ratio of graphene oxide, $(\mathrm{CuPc})$ molecules Fig. 4-8 have two main absorption bands in the visible/near-UV region of the spectrum.

The lower energy band, occurring at around $650 \mathrm{~nm}$ is generally, known as the Q band. In the solid state, these bands are broadened and overlap, so that, phthalocyanines absorb light throughout the entire visible region of the electromagnetic spectrum. It has been suggested that the UV-Vis spectrum of MPcs originates from the molecular orbitals within the aromatic 18 p-electron system and from overlapping orbitals on the central metal and this deal with (Vlaeva et al., 2010).

Figure 5 shows the absorption coefficient. The values of absorption coefficients $(\alpha)$ for all thin films are found to be $>10^{4} \mathrm{~cm}^{-1}$ in the visible region which means that the films have a direct optical energy gap. The variation of the absorption coefficient $(\alpha)$ of $(\mathrm{CuPc})$ as a function of wavelength we notice that that the absorption coefficients values of pure $(\mathrm{CuPc})$ are increasing with the increase of the doping ratio, this is agreement with (AL-Magmaee, 2003).

The change of the extinction coefficient as a function of the wavelength is shown in Fig. 6 for graphene oxide. It can be noted that extinction coefficient has low 

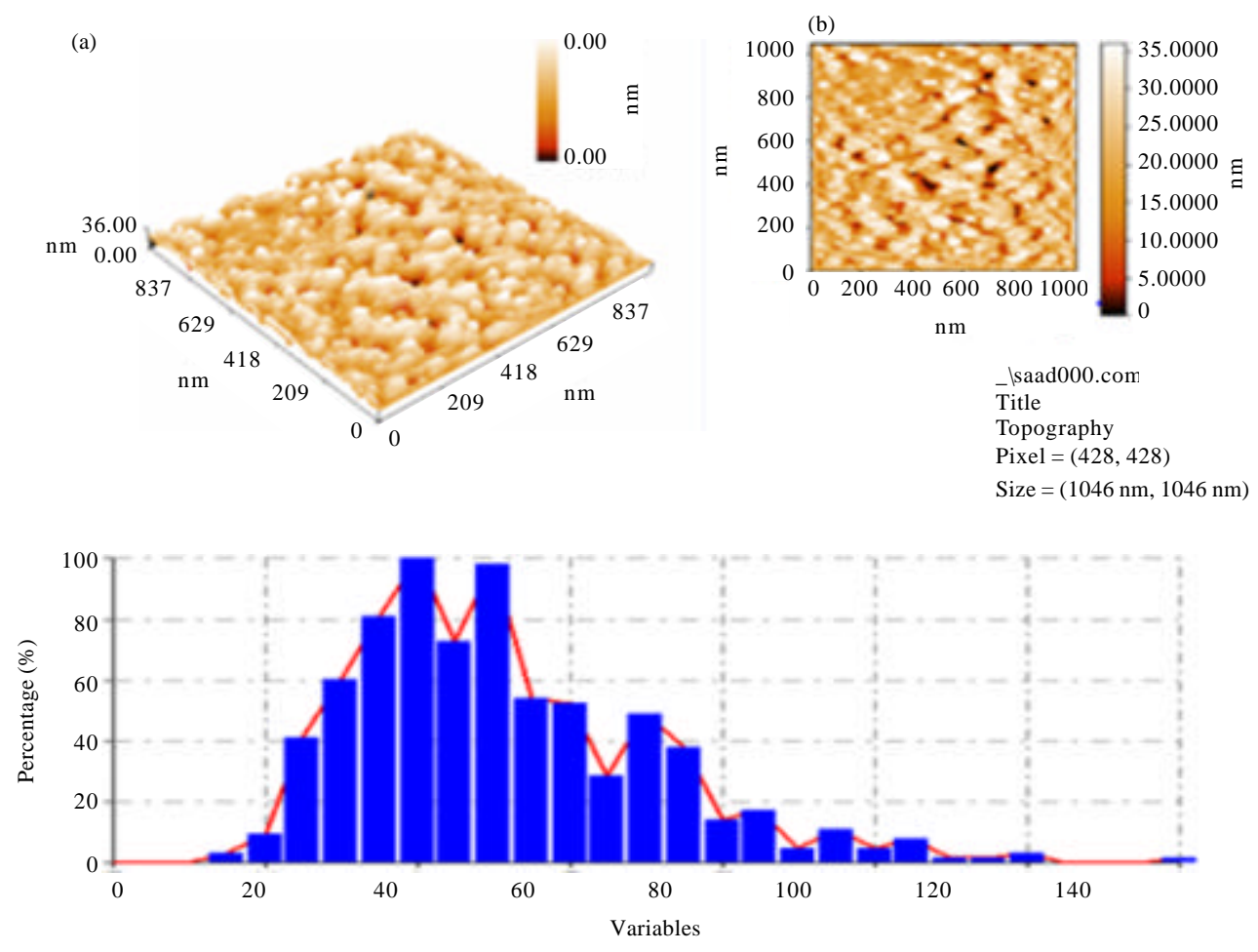

Fig. 3: AFM images of graphene oxide: a) Three-dimensional; b) Two-dimensional and c) Granularity normal distribution chart

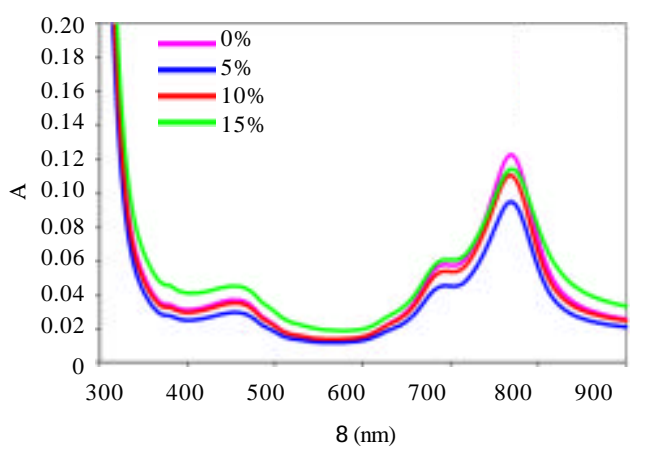

Fig. 4: The absorbance as function of wavelength for different ratio of graphene oxide added to cupper phthalocyanines

value at low concentration but it increases with the increasing of the concentration of graphene oxide. This is attributed to increase absorption coefficient with the increase of weight percentages of graphene oxide. The refractive index. the change of refraction index for graphene oxide ratio as a function of wavelength. The refractive index increases with increasing the weight percentages of the concentration graphene oxide films. The reason of this result is the

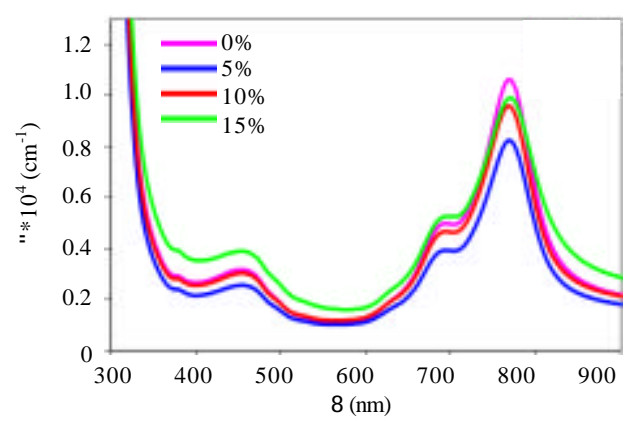

Fig. 5: The absorbance coefficient as function of wavelength for different ratio of graphene oxide added to cupper phthalocyanines

increase of the graphene oxide concentration leads to increase the density of the films and this deal with (Alam et al., 2017). As the ratio of graphene oxide increase the sensitivity for detecting tow type of becteria increase and the highest sensitivity was at the ratio of graphene oxide $15 \%$ (Alam et al., 2017).

Figure 7 shows that the optical energy gap values of pure $(\mathrm{CuPc})$ and its doping are decreasing linearly with the increasing of the concentrations of doping 


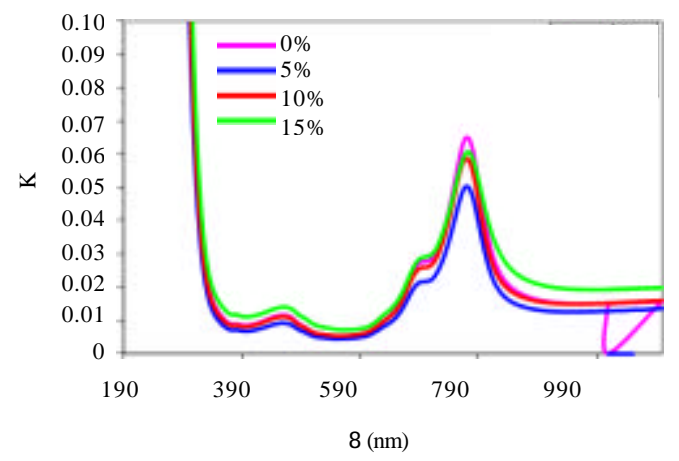

Fig. 6: The extinction coefficient as a function of wavelength for graphene oxide

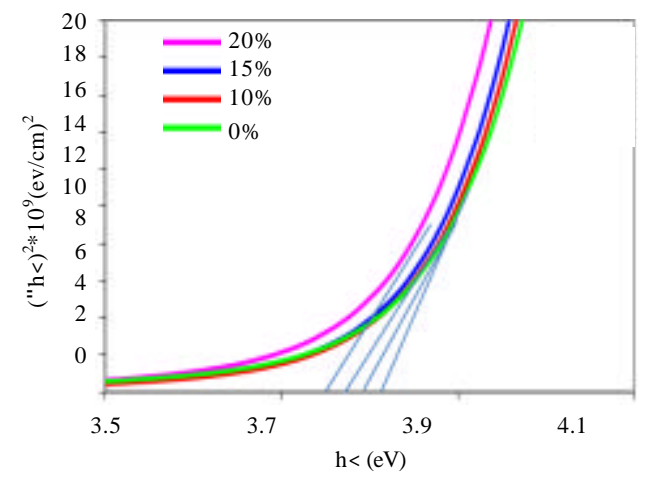

Fig. 7: Relation between $(\alpha \mathrm{hf})^{2}$ versus photon energy

ratio of $\mathrm{GO}$. Where the energy gap for pure $(\mathrm{CuPc})$ films was $3.81 \mathrm{eV}$ and this value decreasing with the increasing of the doping ratio to $3.73 \mathrm{eV}$ at the ratio $20 \%$, this decrease can be explained by the fact that the impurities led to be donor levels inside the energy gap near the conductive band which shifted the fermi level towards the conduction beam (Li et al., 2007).

\section{CONCLUSION}

GO films were successfully prepared via. modified Hummer method preparation of GO films on microscopic morphology. The thickness of the monolayer GO sheet was between $140-150 \mathrm{~nm}$. The presence of oxygen-containing groups and characteristic peaks in FT-IR and XRD analysis further determined the successful preparation of GO sheets. The absorption coefficient increases with increasing of the ratio graphene oxide added to cupper phthaylocinine. The extinction coefficient increases with increasing of the doping ratio of graphene oxide that added to cupper phthaylocinine.

\section{REFERENCES}

AL-Magmaee, Z.T.K., 2003. The study of optical and some electrical properties of Cadmium Oxide $(\mathrm{CdO})$ thin film doped by Silver Oxide $\left(\mathrm{Ag}_{2} \mathrm{O}\right)$ prepared by the chemical spray pyrolysis. MSc Thesis, AL-Mustansiriyah University, Baghdad, Iraq.

Ahn, J.H. and J.H. Je, 2012. Stretchable electronics: Materials, architectures and integrations. J. Phys. D. Appl. Phys., 45: 103001-103001.

Alam, S.N., N. Sharma and L. Kumar, 2017. Synthesis of Graphene Oxide (GO) by modified hummers method and its thermal reduction to obtain Reduced Graphene Oxide (rGO). Graphene, 6: 1-18.

Choi, W. and J.W. Lee, 2012. Graphene: Synthesis and Applications. 1st Edn., CRC Press, Boca Raton, USA., ISBN:9780429105463, Pages: 394.

De La Torre, G., P. Vazquez, F. Agullo-Lopez and T. Torres, 2004. Role of structural factors in the nonlinear optical properties of phthalocyanines and related compounds. Chem. Rev., 104: 3723-3750.

Dimiev, A.M. and J.M. Tour, 2014. Mechanism of graphene oxide formation. ACS. Nano, 8: 3060-3068.

Du, Q., M. Zheng, L. Zhang, Y. Wang and J. Chen et al., 2010. Preparation of functionalized graphene sheets by a low-temperature thermal exfoliation approach and their electrochemical supercapacitive behaviors. Electrochim. Acta, 55: 3897-3903.

Eigler, S. and A. Hirsch, 2014. Chemistry with graphene and graphene oxide-challenges for synthetic chemists. Angew. Chem. Intl. Ed., 53: 7720-7738.

Hsiou, Y.F., Y.J. Yang, L. Stobinski, W. Kuo and C.D. Chen, 2004. Controlled placement and electrical contact properties of individual multiwalled carbon nanotubes on patterned silicon chips. Appl. Phys. Lett., 84: 984-986.

Hummers, Jr. W.S. and R.E. Offeman, 1958. Preparation of graphitic oxide. J. Am. Chem. Soc., 80: 1339-1339.

Kose, S., F. Atay, V. Bilgin and I. Akyuz, 2009. In doped CdO films: Electrical, optical, structural and surface properties. Intl. J. Hydrogen Energy, 34: 5260-5266.

Li, E., A. Kim and L. Zhang, 2007. Modeling excited states of fluorescent compounds with UV-Vis spectra calculations. J. Student Comp. Chem., $1: 1-72$.

Sakamoto, K., E. Okumura and R. Hirohashi, 2004. Phthalocyanines as Functional Dyes. ICP, Tokyo Japan,. 
Shahriary, L. and A.A. Athawale, 2014. Graphene oxide synthesized by using modified hummers approach. Intl. J. Renew. Energy Environ. Eng., 2: 58-63.

Shamaila, S., A.K.L. Sajjad and A. Iqbal, 2016. Modifications in development of graphene oxide synthetic routes. Chem. Eng. J., 294: 458-477.

Spitalsky, Z., M. Danko and J. Mosnacek, 2011. Preparation of functionalized graphene sheets. Curr. Organic Chem., 15: 1133-1150.
Su, C.Y., Y. Xu, W. Zhang, J. Zhao and X. Tang et al., 2009. Electrical and spectroscopic characterizations of ultra-large reduced graphene oxide monolayers. Chem. Mater., 21: 5674-5680.

Vlaeva, I., T. Yovcheva, S. Sainov, V. Dragostinova and S. Stavrev, 2010. Optical properties of PVA films with diamond and Titania nanoparticles. J. Phys. Conf. Ser., 253: 1-6. 